\title{
Nonexponential relaxation and quantum tunnel splitting in the molecular magnet $\mathrm{Fe}_{8}$
}

\author{
Zhi-De Chen ${ }^{1,2}$ and Shun-Qing Shen ${ }^{1}$ \\ ${ }^{1}$ Department of Physics, University of Hong Kong, Pokfulam Rd., Hong Kong \\ ${ }^{2}$ Department of Physics and Institute of Modern Condensed Matter Physics, Guangzhou University, Guangzhou 510405, China
}

(Received 19 September 2002; published 29 January 2003)

\begin{abstract}
Magnetic relaxation in molecular magnets under a sweeping field is studied by taking into account local stray fields. It is found that the randomness of local stray field leads to a distribution of the relaxation rate which subsequently makes the relaxation deviate from the exponential law as predicted by the Landau-Zener model such that the Landau-Zener method needs to be revised to deduce an exact tunneling splitting. The tunneling splitting and distribution width of local stray fields are derived from the experimental data for molecular magnets $\mathrm{Fe}_{8}$.
\end{abstract}

DOI: 10.1103/PhysRevB.67.012408

PACS number(s): 75.45.+j, 75.50.Xx

Magnetic relaxation by quantum tunneling in high spin molecular magnets has become an attractive field of research in recent years. ${ }^{1-15}$ One of the well-studied systems is octanuclear iron (III) oxohydroxo clusters $\mathrm{Fe}_{8}$, which has a welldefined temperature-independent region below $0.36 \mathrm{~K}^{2-6}$ Theoretically the key to understanding magnetic relaxation by the quantum tunneling is the tunnel splitting. The tunnel splitting and the magnetization relaxation are connected via the Landau-Zener model. ${ }^{9,16,17}$ Since the Landau-Zener transition rate is explicitly related to the tunnel splitting, the measurement of the change of the magnetization after one sweep over the resonant point can give the tunnel splitting. ${ }^{17}$ Usually the tunnel splitting is very small (like the groundstate tunneling of $\mathrm{Fe}_{8}$ ), and the transition rate due to tunneling is also very low. One sweep cannot lead to an observable magnetization relaxation. In this case, the tunnel splitting is determined by the Landau-Zener method: ${ }^{3-5}$ multisweeps are done and the tunnel splitting is deduced from the experimental data in a short time region. Up to now, the Landau-Zener method $^{3-5}$ has served as a basic tool to study quantum tunneling in molecular magnets and many other interesting phenomena including the oscillation of tunnel splitting with respect to the field along the hard axis and the parity effect for odd and even resonance. . $^{3,19}$ The tunnel splitting from the Landau-Zener method is found to be sweeping-rate independent and agrees with the result found by using a square-root decay method. ${ }^{3-5}$ However, there is still a puzzle that magnetization relaxation under a sweeping field shows a clear deviation from the exponential behavior as predicted by the Landau-Zener model. ${ }^{4,5}$ Such a consequence may lead to a question of how the tunnel splitting is deduced from the experimental data in short time region exactly. This is the main motivation of the present paper.

In the present paper, we start with the biaxial spin model with a local stray field to study the magnetization relaxation behaviors. It is shown that the "uncompensated" transverse component of the local stray field leads to a distribution of relaxation rates which makes the relaxation in molecular magnets follow a different mechanism as in some complex systems like spin glass. ${ }^{20,21}$ Our analysis shows that the relaxation is determined by two independent quantities: the tunnel splitting and the distribution width of the local stray field. Although the magnetization relaxation deviates the ex- ponential law we can derive the two quantities from experimental data of molecular magnets $\mathrm{Fe}_{8}$ successfully.

The biaxial spin model for the molecular magnets $\mathrm{Fe}_{8}$ with a local stray field is written as ${ }^{18,19,22}$

$$
H=K_{1} S_{z}^{2}+K_{2} S_{y}^{2}-g \mu_{B} \mathbf{S} \cdot(\mathbf{B}+\mathbf{h}),
$$

where $K_{1}>K_{2}>0$, and $\mathbf{B}$ is the applied magnetic field. $\mathbf{h}$ is the local stray field which may originate from the interactions between the giant spin and the environmental spins (including other giant spins or nuclear spins). To simplify the problem we assume that $\mathbf{h}$ has a Gaussian distribution with an equal distribution width in all directions, ${ }^{23}$

$$
P(\mathbf{h})=\frac{1}{\left(2 \pi \sigma^{2}\right)^{3 / 2}} \exp \left[-\left(\mathbf{h}-\mathbf{h}_{0}\right)^{2} / 2 \sigma^{2}\right] .
$$

Since our main interest is the magnetization relaxation under a sweeping field, the external magnetic field is taken to be $\mathbf{B}=\left\{B_{x}, 0,0\right\}: B_{x}=n \Delta B \pm c t$ where $n$ is integer, $\Delta B$ is the field interval between neighboring resonant tunneling, and $c=d B_{x} / d t$. In the following calculation, we take $K_{1}$ $=0.321 \mathrm{~K}, K_{2}=0.229 \mathrm{~K}$ for the molecular magnets $\mathrm{Fe}_{8} \cdot{ }^{3}$ When the field along the easy axis is sweeping over the resonant field $n \Delta B$, the biased local stray field $h_{x}$ will be compensated by the sweeping field which brings spins into the resonant tunneling and leads to a continuous relaxation. If we omit the transverse component of the local stray field, then all the spins will have the same tunneling rate inside the resonant window. The resulted relaxation is the simple exponential decay according to the Landau-Zener model, ${ }^{4,5}$

$$
M(t)=M_{0} e^{-\Gamma t},
$$

where $\Gamma=k P_{L Z} c / A$ and $P_{L Z}$ is the Landau-Zener transition rate, $P_{L Z}=1-\exp \left(-\pi \Delta_{n}^{2} / \nu_{n} c\right), \Delta_{n}$ is the tunnel splitting, $\nu_{n}$ $=2 g \mu_{B} \hbar(2 s-n), k=2$ for $n=0, k=1$ for $n=1,2,3, \ldots$, and $A$ is the amplitude of the ac field used in the experiment. In the low transition rate limit, i.e., $P_{L Z} \ll 1$, which holds for the $\mathrm{Fe}_{8}$ system, ${ }^{4,5}$ the above equation leads to

$$
\ln \frac{M(t)}{M_{0}}=-\frac{k \pi}{\nu_{n} A} \Delta_{n}^{2} t+\frac{1}{2}\left(\frac{\pi \Delta_{n}}{\gamma_{n} c}\right)^{2} \frac{k c}{A} t+\cdots
$$


In the Taylor series expansion, one has

$$
\frac{M_{0}-M(t)}{M_{0}}=\frac{k \pi t}{\nu_{n} A} \Delta_{n}^{2}-\frac{1}{2}\left[\left(\frac{k \pi t}{\nu_{n} A}\right)^{2}+\frac{\pi^{2} k t}{\gamma_{n}^{2} c A}\right] \Delta_{n}^{4}+\cdots .
$$

In a short time region, we can keep the first term on the right side of Eq. (4), and then the tunnel splitting $\Delta_{n}$ can be deduced from the magnetization relaxation of the molecular magnets.

However, the tunnel splitting strongly depends on the strength of the transverse local stray field. In other words, the local stray field will leads to a distribution of the tunneling splitting, and furthermore a distribution of the relaxation rates. Consequently, the resulted relaxation is modified as

$$
M(t)=M_{0} \int d \mathbf{h} P(\mathbf{h}) e^{-\Gamma(\mathbf{h}) t},
$$

where

$$
\Gamma(\mathbf{h})=\left\{1-\exp \left[-\pi \Delta_{n}^{2}(\mathbf{h}) / \nu_{n} c\right]\right\} k c / A .
$$

The tunnel splitting $\Delta_{n}^{2}(\mathbf{h})$ can be calculated by the instanton method, ${ }^{19,23}$

$$
\Delta_{n}^{2}(\mathbf{h}) \simeq \gamma_{ \pm}(\mathbf{h}) \Delta_{n 0}^{2},
$$

where the renormalized factor is caused by the local stray field, $\gamma_{ \pm}(\mathbf{h})=\left[\cosh \left(2 q h_{y}\right) \pm \cos \left(2 d_{n} h_{z}\right)\right] / 2$ and \pm stands for the even and odd resonant tunneling.

$$
d_{n}=\frac{g \mu_{B}}{2 K_{1}} \int_{0}^{\pi} \frac{d \phi}{1-\frac{K_{2}}{K_{1}} \sin ^{2} \phi-\frac{g \mu_{B} n \Delta B}{2 s K_{2}} \cos \phi},
$$

$q=g \mu_{B} \pi / 2\left(K_{1} K_{2}-K_{2}^{2}\right)^{1 / 2}$, and $\Delta_{n 0}$ is independent of the transverse field. In the low transition rate limit, ${ }^{4,5}$ we have

$$
M(t) \simeq M_{0} \int d \mathbf{h} P(\mathbf{h}) \exp \left\{-\gamma_{ \pm}(\mathbf{h}) \Gamma_{0} t\right\}
$$

where $\Gamma_{0}=k \pi \Delta_{n 0}^{2} /\left(\nu_{n} A\right)$.

The above equation shows that, in the low transition rate limit, the magnetic relaxation is sweeping-rate independent, provided that the sweeping rate is large enough, namely, for $d B_{x} / d t \gtrsim 1.0 \mathrm{mT} / \mathrm{s} .{ }^{4,23}$ In the absence of the transverse local stray field, i.e., $P(\mathbf{h})=\delta(\mathbf{h})$, we have $M(t) \simeq M_{0} e^{-\Gamma_{0} t}$ for an even resonant tunneling and $M(t)=M_{0}$ for an odd resonant tunneling as expected. The effect of the transverse local stray field can be observed by doing the integration in Eq. (9). Numerical results ${ }^{24}$ as shown in Fig. 1 show that the magnetization relaxation deviates apparently from the exponential decay as the distribution width becomes larger than $0.03 \mathrm{~T}$, which is in qualitative agreement with the experimental measurement. The nonexponential decay indicates the LandauZener model cannot be applied to measure the tunneling splitting explicitly. In fact, for a system with a distribution of relaxation time, the resulted relaxation can be a large variety of shapes of decay. ${ }^{20,21}$ The above analysis shows that we cannot deduce the tunnel splitting according to Eq. (4) when

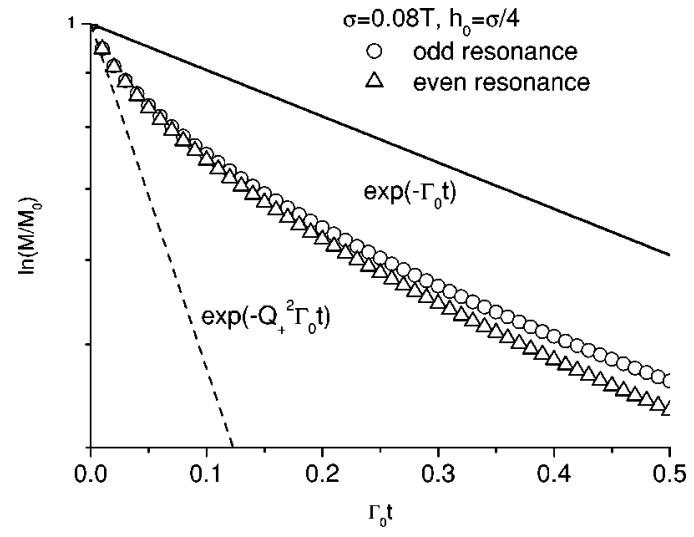

FIG. 1. Short time relaxation behavior for both odd and even resonant tunneling with $\sigma=0.08 \mathrm{~T}$ and $h_{0}=\sigma / 4$.

the relaxation deviates strongly from the exponential law. On the other hand, consider the local stray fields one obtains

$$
\frac{M_{0}-M(t)}{M_{0}}=\frac{k \pi t}{\nu_{n} A}\left\langle\Delta_{n}^{2}\right\rangle-\frac{1}{2}\left[\left(\frac{k \pi t}{\nu_{n} A}\right)^{2}+\frac{\pi^{2} k t}{\gamma_{n}^{2} c A}\right]\left\langle\Delta_{n}^{4}\right\rangle+\cdots
$$

where $\langle X\rangle=\int d \mathbf{h} P(\mathbf{h}) X$. Comparing with Eq. (4), one can see that the tunnel splitting determined from $[M(t)$ $\left.-M_{0}\right] / M_{0}$ under such an approximation is $\sqrt{\left\langle\Delta_{n}^{2}\right\rangle}$ instead of $\Delta_{n}$. From Eq. (7), one has

$$
\sqrt{\left\langle\Delta_{n}^{2}\right\rangle}=Q_{ \pm} \Delta_{n 0}
$$

where the averaging renormalization factor due to the local stray fields is

$$
Q_{ \pm}=\frac{1}{\sqrt{2}}\left[e^{2 q^{2} \sigma^{2}} \cosh \left(2 q h_{0}\right) \pm e^{-2 d_{n}^{2} \sigma} \cos \left(d_{n} h_{0}\right)\right]^{1 / 2}
$$

Dependence of $Q_{ \pm}$on the distribution width $\sigma$ is plotted in Fig. 2. It is seen that $Q_{ \pm}$increase rapidly with increasing $\sigma$

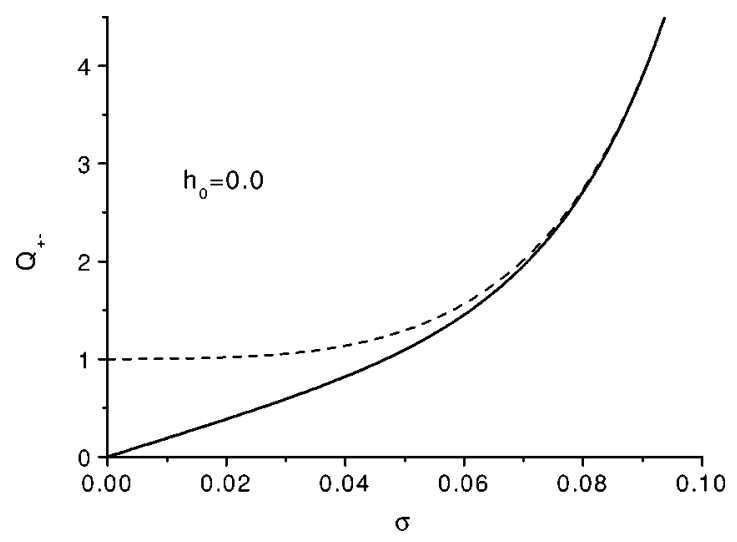

FIG. 2. Illustration of the dependence of $Q_{ \pm}$on the distribution width $\sigma$. The dashed and solid lines represent for $Q_{+}$and $Q_{-}$, respectively. 


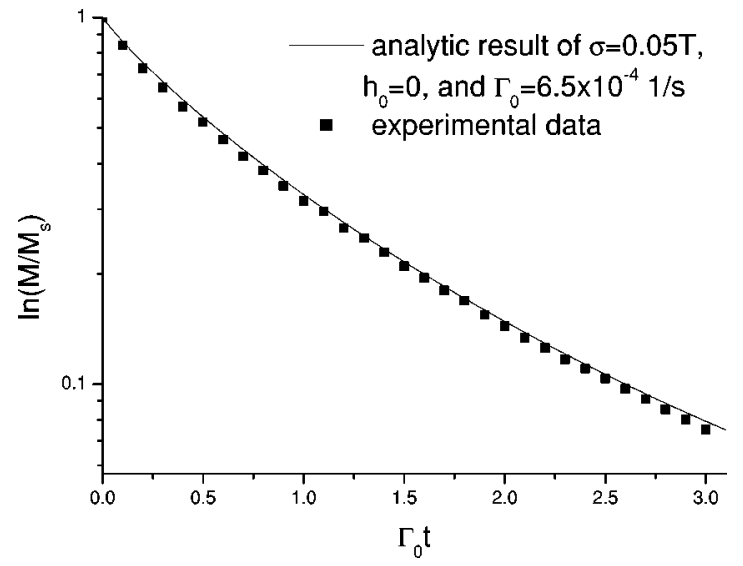

FIG. 3. Relaxation curve of ground state tunneling in the $\mathrm{Fe}_{8}$ molecule using a sweeping field with $A=7.2495 \times 10^{-2} \mathrm{~T}$ and $d B_{x} / d t=1.4 \mathrm{mT} / \mathrm{s}$.

and become coincident as $\sigma>0.08 \mathrm{~T}$. For an even resonance, $Q_{+} \geqslant 1$, and $\sqrt{\left\langle\Delta_{n}^{2}\right\rangle}$ is always larger than the exact tunnel splitting $\Delta_{n 0}$, while for an odd resonance, the tunnel splitting $\sqrt{\left\langle\Delta_{n}^{2}\right\rangle}$ is not always quenched once the local stray field appears.

Now we are ready to derive the tunneling splitting from the experimental data of $\mathrm{Fe}_{8}$. The experimental data of the ground-state tunneling in $\mathrm{Fe}_{8}$ were provided by Wernsdorfer. $^{4,5}$ To fit the experimental data we choose $\Gamma_{0}$ $=6.5 \times 10^{-4} / \mathrm{sec}$ and $\sigma=0.05 \mathrm{~T}$. Both theoretical and experimental results are plotted in Fig. 3. One can find that our analytic result fits the experimental curve quite well. The estimated value $\sigma=0.05 \mathrm{~T}$ is consistent with the linewidth of the resonance for $\mathrm{Fe}_{8} \cdot{ }^{8}$ However, the hole-digging method ${ }^{5,6}$ gives a value of $0.03 \mathrm{~T}$, which is smaller than what we estimated. The tunnel splitting from the chosen $\Gamma_{0}$ is $\Delta_{0}=7.85$ $\times 10^{-8} \mathrm{~K}$, and the renormalization factor is $Q_{+}(\sigma$ $=0.05 \mathrm{~T}) \simeq 1.292$. Therefore we have $\sqrt{\left\langle\Delta_{0}^{2}\right\rangle} \simeq 1.01$ $\times 10^{-7} \mathrm{~K}$, which is very closed to $1.0 \times 10^{-7} \mathrm{~K}$ in the conventional Landau-Zener model by Wernsdorfer et al. ${ }^{4,5}$ So what measured in the Landau-Zener model is $\sqrt{\left\langle\Delta_{0}^{2}\right\rangle}$ instead of $\Delta_{0}$ in the short time limit. Clearly, $\Delta_{0}$ and $\sqrt{\left\langle\Delta_{0}^{2}\right\rangle}$ are two different concepts. For $\mathrm{Fe}_{8}$, since the averaging renormalization factor is as large as 1.292 , we should consider the effect from the local stray field. Our result shows that the magnetization relaxation is determined by the tunnel splitting and the distribution width of the local stray field. The later quantity leads to the relaxation deviating from the exponential decay. On the other hand, after the local stray field is introduced, the main modification to the kinetic equation of the relaxation is to replace $\Delta_{n 0}$ with $\sqrt{\left\langle\Delta_{n}^{2}\right\rangle}$ in the low transition rate limit.

It should be noted that the tunnel splitting from the experimental data is sweeping-rate independent since the magnetic relaxation is independent of the sweeping rate when $c$ $>1.0 \mathrm{mT} / \mathrm{sec}$. From Eq. (6) we found that, rigorously speaking, the magnetization relaxation depends on the sweeping rate $c$ via the expression for $\Gamma(\mathbf{h})$. However, the larger $c$ leads to a smaller transition rate. When the low transition

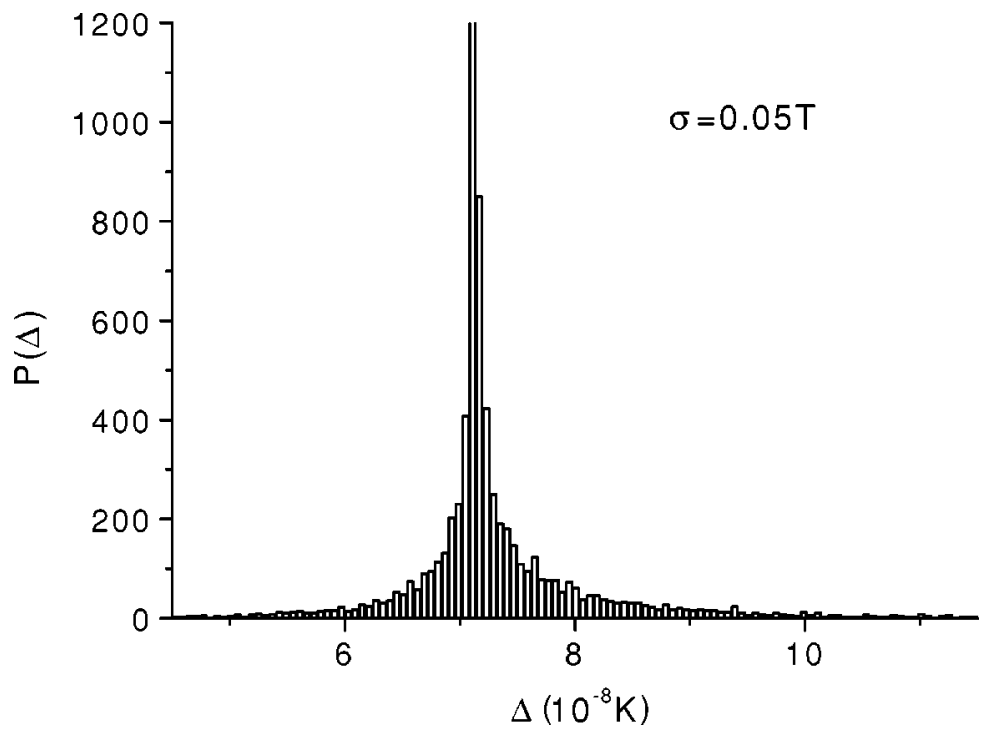

FIG. 4. Distribution of ground-state tunnel splitting for a $\mathrm{Fe}_{8}$ model with higher-order term $C\left(S_{+}^{4}+S_{-}^{4}\right)$ in Hamiltonian under a local stray field with a Gaussian distribution. $(C=-2.95$ $\left.\times 10^{-5} \mathrm{~K}\right)$.

rate approximation becomes valid for a sufficiently large $c$, the transition rate is independent of $c$. This is consistent with the experimental measurement. Another problem is that the above analysis is based on the adiabatic approximation. It is assumed that all the spins have sufficient time to tunnel no matter how small the tunnel splitting is. When the distribution width of the local stray field is taken into account, the distribution of tunnel splitting becomes quite large. Approximately there will be a cutoff tunnel splitting $\Delta_{n c} \simeq \nu_{n} c / \pi$ such that the spins with the tunnel splitting lower than $\Delta_{n c}$ will have no sufficient time to tunnel. ${ }^{15,25}$ To find out the distribution of the tunnel splitting due to the local stray field, we have made Monte Carlo simulation for $\mathrm{Fe}_{8}$ systems with higher-order terms in the Hamiltonian. The resulted distribution of tunnel splitting is plotted in Fig. 4 for $\sigma=0.05 \mathrm{~T}$. The tunnel splitting spreads over about 2 to 3 orders which is much narrower than that in $\mathrm{Mn}_{12}$ molecules due to dislocation. ${ }^{15,25}$ As the local stray field from dipolar-dipolar or hyperfine interaction in $\mathrm{Mn}_{12}$ is stronger than that in $\mathrm{Fe}_{8}$ molecules and dislocation will lead to a wider distribution of the tunnel splitting. The $\mathrm{Mn}_{12}$ system will have a much wider distribution of relaxation time than $\mathrm{Fe}_{8}$ system. It implies that the magnetization relaxation in $\mathrm{Mn}_{12}$ system will deviate far away from the exponential decay.

In conclusion we have studied the effect of the local stray field on the magnetic relaxation under a sweeping field in $\mathrm{Fe}_{8}$ molecules. The uncompensated transverse local stray field leads to a distribution of the transition rate such that the relaxation deviates from the exponential law. The interplay of the quantum tunneling and the distribution of the local stray field determines the magnetization relaxation. Based on this picture we proposed a revised scheme to deduce the tunnel splitting $\Delta_{n}$ from experimental measurement instead 
of the conventional Landau-Zener method. Our conclusion can be generalized to other molecular magnets since the local stray field due to the dipolar-dipolar or hyperfine interaction exists extensively.
The authors thank W. Wernsdorfor for his providing the experimental data in Fig. 3. The work was supported by a grant from the Research Grants Council of Hong Kong, China, and a CRCG grant of the University of Hong Kong.
${ }^{1}$ L. Gunther and B. Barbara, Quantum Tunnelling of Magnetization-QTM'94 (Kluwer, Dordrecht, 1995).

${ }^{2}$ C. Sangregorio, T. Ohm, C. Paulsen, R. Sessoli, and D. Gatteschi, Phys. Rev. Lett. 78, 4645 (1998).

${ }^{3}$ W. Wernsdorfer and R. Sessoli, Science 284, 133 (1999).

${ }^{4}$ W. Wernsdorfer, R. Sessoli, A. Caneschi, D. Gatteschi, A. Cornia, and D. Mailly, J. Appl. Phys. 87, 5481 (2000).

${ }^{5}$ W. Wernsdorfer, Adv. Chem. Phys. 118, 99 (2001).

${ }^{6}$ W. Wernsdorfer, T. Ohm, C. Sangregorio, R. Sessoli, D. Mailly, and C. Paulsen, Phys. Rev. Lett. 82, 3903 (1999).

${ }^{7}$ W. Wernsdorfer, R. Sessoli, and D. Gatteschi, Europhys. Lett. 47, 254 (1999).

${ }^{8}$ N.V. Prokof'ev and P.C.E. Stamp, Phys. Rev. Lett. 80, 5794 (1998).

${ }^{9}$ B. Barbara, L. Thomas, F. Lointi, I. Chiorescu, and A. Sulpice, J. Magn. Magn. Mater. 200, 167 (1999).

${ }^{10}$ T. Ohm, C. Sangregori, and C. Paulsen, Eur. Phys. J. B 6, 195 (1998); J. Low Temp. Phys. 113, 1141 (1998).

${ }^{11}$ A. Cuccoli, A. Fort, A. Rettori, E. Adam, and J. Villain, Eur. Phys. J. B 12, 39 (1999).

${ }^{12}$ W. Wernsdorfer, A. Caneschi, R. Sessoli, D. Gatteschi, A. Cornia, V. Villar, and C. Paulsen, Phys. Rev. Lett. 84, 2965 (2000).

${ }^{13}$ E.M. Chudnovsky, Phys. Rev. Lett. 84, 5676 (2000); N.V. Prokof'ev and P.C.E. Stamp, ibid. 84, 5677 (2000).

${ }^{14}$ J.Q. Liang, H.J.W. Müller-Kirsten, D.K. Park, and F.C. Pu, Phys. Rev. B 61, 8856 (2000); Y.H. Jin, Y.H. Nie, J.Q. Liang, Z.D.
Chen, W.F. Xie, and F.C. Pu, ibid. 62, 3316 (2000).

${ }^{15}$ E.M. Chudnovsky and D.A. Garanin, Phys. Rev. Lett. 87, 187203 (2001); D.A. Garanin and E.M. Chudnovsky, Phys. Rev. B 65, 094423 (2002).

${ }^{16}$ L. Landau, Phys. Z. Sowjetunion 2, 46 (1932); C. Zener, Proc. R. Soc. London, Ser. A 137, 696 (1932).

${ }^{17}$ S. Miyashita, J. Phys. Soc. Jpn. 64, 3207 (1995); 65, 2734 (1996).

${ }^{18}$ A. Garg, Europhys. Lett. 22, 205 (1993).

${ }^{19}$ Z.D. Chen, Phys. Rev. B 65, 085313 (2002).

${ }^{20}$ R.G. Palmer, D.L. Stein, E. Abrahams, and P.W. Anderson, Phys. Rev. Lett. 53, 958 (1984).

${ }^{21}$ P. Erhart, A.M. Portis, B. Senning, and F. Waldner, J. Phys.: Condens. Matter 6, 2881 (1994); P. Erhart, B. Senning, and F. Waldner, ibid. 6, 2893 (1994).

${ }^{22}$ E.M. Chudnovsky and L. Gunther, Phys. Rev. Lett. 60, 661 (1988).

${ }^{23}$ Z.D. Chen, J.-Q. Liang, and S.Q. Shen, Phys. Rev. B 66, 092401 (2002).

${ }^{24}$ The numerical result shows that the resulted relaxation curve is not sensitive to the distribution center. In the present paper, we present results for $h_{0}=0$ or $h_{0}=\sigma / 4$ according to the experimental result (Ref. 10).

${ }^{25}$ K.M. Mertes, Y. Suzuki, M.P. Sarachik, Y. Paltiel, H. Shtrikman, E. Zeldov, E. Rumberger, D.N. Hendrickson, and G. Christou, Phys. Rev. Lett. 87, 227205 (2001). 